\title{
Developing a composite based elliptic spring for automotive applications
}

\begin{abstract}
An automotive suspension system is designed to provide both safety and comfort for the vehicle occupants. In this study, finite element models were developed to optimize the material and geometry of the composite elliptical spring based on the spring rate, log life and shear stress parameters. The influence of the ellipticity ratio on the performance of woven rovingï wrapped composite elliptical springs was investigated both experimentally and numerically. The study demonstrated that composite elliptical springs can be used for light and heavy trucks with substantial weight reduction. The results showed that the ellipticity ratio significantly influenced the design parameters. Composite elliptic springs with ellipticity ratios of $\mathrm{a} / \mathrm{b}=2$ had the optimum spring parameters.
\end{abstract}

Keyword: Finite element models; Composite elliptical spring; Automotive applications 